\title{
IoT and Wireless Sensor Network for Interactive Waka Structure
}

\author{
A. Gaddam, K.O. Lundqvist \\ School of Engineering and Computer Science \\ Victoria University of Wellington \\ Wellington, New Zealand \\ Anuroop.Gaddam@vuw.ac.nz
}

\author{
J. Citizen ${ }^{*}$, D.B. Calixto ${ }^{\dagger}$ \\ *School of Media Arts \\ †Centre for Engineering \& Industrial Design \\ Waikato Institute of Technology \\ Hamilton, New Zealand \\ Joe.Citizen@wintec.ac.nz
}

\begin{abstract}
Partnership between technologists, engineers and artists often produces new forms of interactive and complex artworks. This paper discusses the role of a smart interactive art structure and reviews the novel ways in which it can serve the intended purpose. The interactive waka project is a multidisciplinary project which involves in the production of an interactive six-meter-tall COR-TEN plate steel waka (traditional canoe) sculpture informed by Mātauranga Māori (Māori knowledge) about the Matariki star cluster. (Pleiades). This smart interactive structure will be will be located besides the Waikato river in Hamilton, New Zealand. The waka sculpture as a smart structure serves several main interrelating purposes that are centred on its symbolic, architectonic, and interactive functions. The use of human and environmental monitoring will enable coconstitutive real-time interactions, which will themselves be performatively incorporated into the field of relations. All the information gathered from the wireless sensors will be sent to the cloud, where it will be processed to determine how the waka structure's light and sound patterns should behave. The waka structure will be self-contained in terms of power, using sustainable energy sources such as solar, and will not be active unless humans are present. Data collected through the environmental wireless sensors network will be publically available through a website, using an open-source platform and the smart interactive structure will be gifted to the City of Hamilton in New Zealand.
\end{abstract}

Keywords- smart interactive art; wireless sensor network; internet of things; environmental monitoring; architectonic;

\section{INTRODUCTION}

Embedded systems and wireless sensor networks based interaction is becoming a popular phenomenon for many artistic installations now a day. Modern artists have been particularly captivated in audience involvement with the artworks [1]. An artwork which is capable of interactions could potentially transform its viewers into participants. Recent advancements in digital technology, embedded wireless sensor systems have magnified the widespread technology access, thereby enabling the development of interactive systems in sound, light and multimedia based installations of art [2]. He waka eke noa ('a boat we all on together') refers to the research partnership that seeks to create a public artwork tentatively called 'The Matariki Interactive Waka Project'. Informed by an environmental wireless sensor network, it will be built of COR-TEN plate steel, interactive LED lighting, and 5.1 surround sounds. It aims to raise awareness of the interconnected nature of the Waikato river - in its physical, spiritual, historical, and mental capacities through the movements, vibrations and interactions of human and non-human participants (such as animals, the weather, the seasons, phases of the moon, plant growth, the river itself etc.) It will do this by singly accessing one of the seven identified Matariki stars as different 'modes' to emphasize different aspects of the river's holistic environment. Similar to our approach is a project which used sensing technology in art installations, by using wireless sensor based modules on an art installation, Rasamimanana et al. were able to created musical sounds when the art structure was interacted [3]. The interactive Audience Sensor Kit (TASK) is another related project when the technology is combined with the art installations. By using different types of sensor this module can trace the movements of its audience and react according to their movements. In [4] and [5] the art installations create an interactive experience to the audience around thereby generated an immersive experience. Also, this type of merging digital technology with the artistic considerations will draws the general audience towards the modern sensor technology.

\section{The Purpose of The WaKa Structure}

The aim of this project is that movements of people and animals around the interactive art structure will modify aspects of each 'Matariki star mode' which are themselves determined by seasonal and environmental conditions of the day. By focusing the attention on each star's domain, different modes of lights up its LED matrix. Each star mode is informed by live environmental data from their relevant domain, shown as a simplified animated gif on the LED matrix. Also, the movement of people will be shown in real-time as sparks of light moving across each LED display. This interactive art structure serves several main interrelating purposes that are centred on its symbolic, architectonic, and interactive functions.

\section{A. Symbolic Function of the Interactive Waka Structure}

The waka structure itself acts a symbolic function, the structure connotes a boat in form. A waka is fundamentally a vessel, in all its metaphorical as well as literal forms: it references travelling, journeys, and the craft by which these are 
accomplished. It is a structure made specifically for travelling in an environment that decentres the pre-eminence of people, it celebrates the creatures and food found at in the river, and highlights the site as a meeting place between cultures, ideas, and trade, across the ebb and flow of space and time. The individual Matariki stars function as access points to different domains of the natural world in a more interconnected way than typical western conceptions of it, which typically frame 'things' and 'people' as being epistemically discrete Figure 1 below show the artistic illustration of the proposed interactive waka structure.
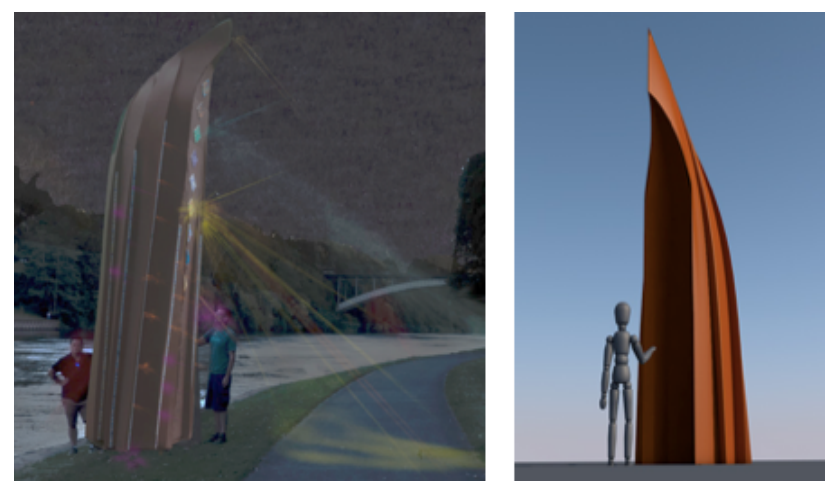

Figure 1. Artist illustations of interactive waka structure on the bank of river.

The Lighting and LED panels on the waka structure are not simply 'nice to have' feature - instead they are core integrated aspects of the interactive structure. The structure has to operate so that every aspect of it highlights interconnection with the universe in an interactive manner. The 'art' is not made from the structure itself, but from the interactions of participants (both human and non-human) with it and each other - the art is mutually co-constituted and continuously emergent. Each 'rib' of the waka will also have LEDs and motion sensors, with the potential to have additional animations on these ribs. The structure will be designed to limit the light spill, and help shape the light beams. The placement of the RGB modules or strips is critical as they cannot be too centred or too much towards the edge, so that their light throws only graze the curve in front of them, and no further. A Musical Instrument Digital Interface (MIDI) based communications protocol, digital interface will be installed on the interactive structure. This will enable the usage of a wide variety of digital music instruments, different types of audio devices to connect and communicate with one another, so that localised feedback between participants and the other modifiers will be enabled. The motion sensors on the structure will also activate digital MIDI instrument sounds. The idea is to have the capacity to activate sound and lighting during the dusk, night, and dawn times, but only sounds can be activated during the daytime. The LED panels relate directly to each of the Matariki stars and their individual domains as determined by the types of sensors being used, and the movements of those travelling around the outside of the waka itself.

\section{B. Architectonic Function of the Interactive Waka Structure}

The placement and orientation of the waka at the selected site as shown in figure 2 below with red coloured marker, is very important as it will stage and frame encounters for human participants as they travel down the river path from the north or south. It will be seen from two traffic bridges, and from a memorial park located across the river.

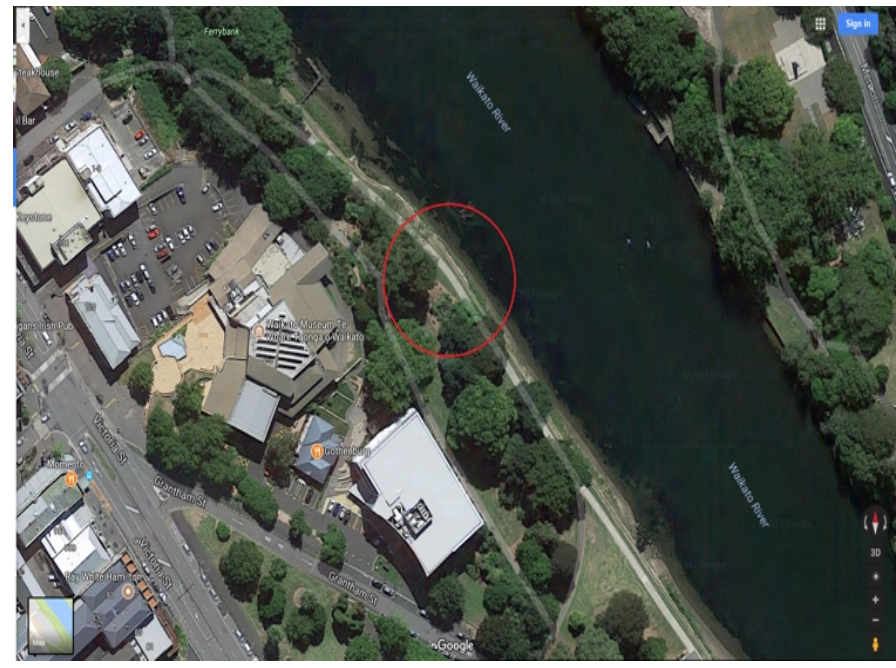

Figure 2. Site location, image taken from google maps.

The structure needs to shelter to some extent the sensors, lighting and sound aspects from the environment, but also enable their operation. Different types of digital sounds will be used, heard from a marine-grade sub-amplifier and woofer suspended from within the structure. The rib lighting will use minimalistic RGB dot modules recessed behind symbolic patterns cut out of the steel using an industrial weter-jet cutting process. The top of the structure will have a top-end image projector emplaced to that its beam hits the ground at an angle near the river's edge. The rib lighting, LED panels matrices and waka sensors need to be incorporated into the structure to enable wiring to puncture the skin of the waka in a minimal manner - it may be that wiring is external to display its own construction, which is dependent on wired connections with the microcontrollers used and their power supply modules.

\section{Interactivity Function of the Interactive Waka Structure}

Parallel to the RGB matrix or strips on the ribs of the waka structure, there will be different types of thermal imaging camera sensors. These camera sensors will enable sensing to occur in both the $x$ and $y$ planes in a linear manner, so that the whole when seen from above take on a radial form. As each rib is relatively narrow $(210 \mathrm{~mm}$ at the base) compared to its curvature (approx. $510 \mathrm{~mm}$ at the base) the sensing capabilities will occur within narrow zones of sensitivity. In order to achieve this, it will be essential to ensure that the camera sensors are placed towards the outside rather than the inside. Having sensors stacked in a vertical manner in each rib will enable sensing to occur in space not occupied by humans - its intended that birds and other warm-blooded animals (non-human) will also be able to trigger interactions. At the apex of the underside of the front arch of the structure, there will be more wireless sensors, a Raspberry Pi with a NoIR camera, which will need to hang straight down so that their sensing capabilities incorporate the LED panel zone below. The very topmost tip of the waka an aerial will installed for wireless communication to the base station. 


\section{WAKA INTERACTIVE STRUCTURE'S WIRELESS SENSOR NETWORK DESIGN}

A The wireless sensor network is designed to have multiple types of sensors installed in and around the structure. These sensors are responsible in detecting both human and non-human interaction with the waka. All the sensors will be connected to Arduino based microcontroller. A combination of ZigBee and Wi-Fi based sensors will be used to create a network for the data transmissions between the sensors and the cloud (internet) as shown in block diagram below in figure 3 .

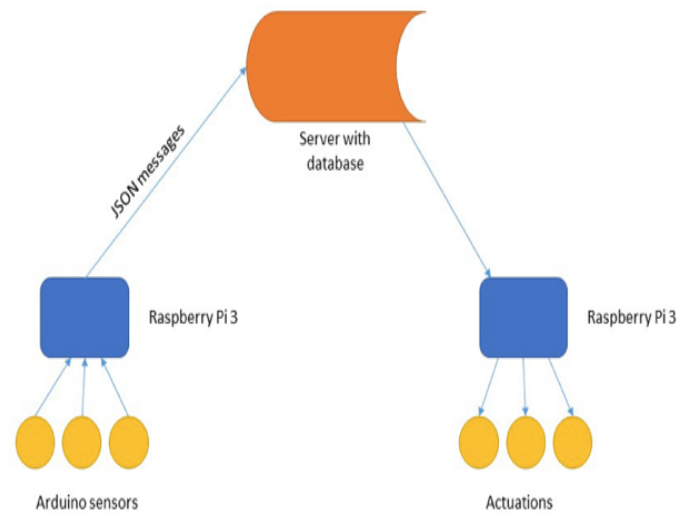

Figure 3. Block diagram of Waka structure's wireless sensor network

The data collected by the sensors will be pre-processed within the sensor's level and then the data will be transmitted to a Raspberry Pi module connected to a dedicated cloud. Based on the data collected on the cloud, the Raspberry Pi module will signal will be sent back to the structure enabling it to project a specified light pattern or sound, as illustrated below in figure 4 .

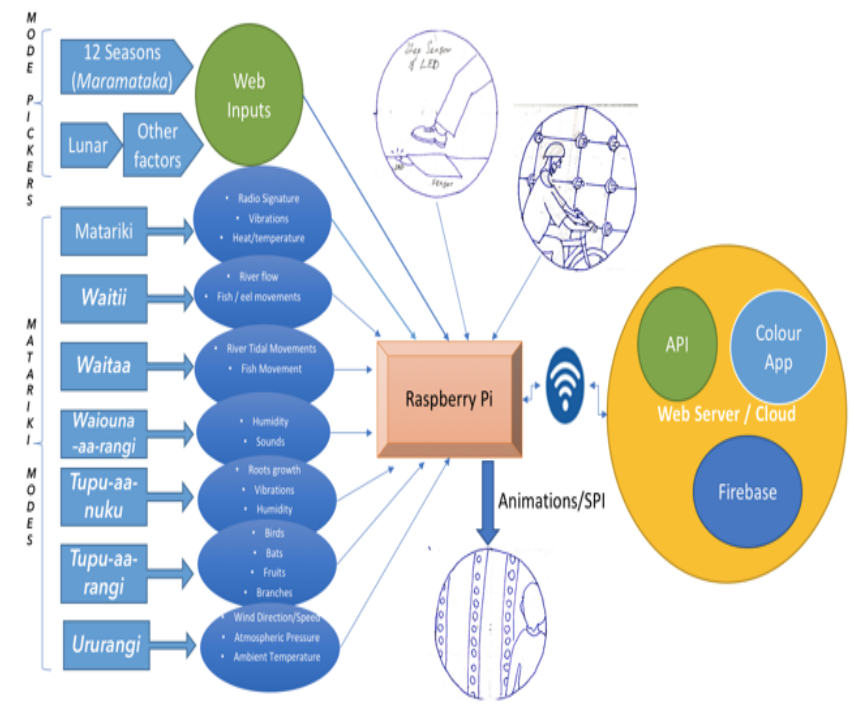

Figure 4. Proposed design and relavent measurements to be used in relation to each of the Matariki stars and their domains

\section{A. Sensors \& Environmental Variables}

In accordance to the requirements of the project, the following sensors shown below in table 1, will be used to monitor various environmental variables that will be interacting with the Waka structure.
TABLE I. PROPOSED SENSORS AND MEASUREMENTS TO BE USED IN RELATION TO EACH OF THE MATARIKI STARS AND THEIR DOMAINS

\begin{tabular}{|c|c|c|}
\hline Star & Domain & Sensors / measurements \\
\hline Matariki & $\begin{array}{l}\text { Interconnectedness } \\
\text { with the } \\
\text { environment }\end{array}$ & $\begin{array}{l}\text { - Radio signature of Matariki star } \\
\text { - Thermopile and ultrasonic sensors } \\
\text { (heat and ultrasound) for motion } \\
\text { tracking on the waka sculpture itself } \\
\text { - Radio signatures of mobile phones } \\
\text { - Traffic vibrations from nearby } \\
\text { bridge(s) }\end{array}$ \\
\hline Waitii & Fresh water & $\begin{array}{l}\text { - Tracking the movements of fish, eels, } \\
\text { koura etc. } \\
\text { - Also, the river: Currents, speed, } \\
\text { volume, temperature, turbidity } \\
\text { (clearness) }\end{array}$ \\
\hline Waitaa & Salt water & $\begin{array}{l}\text { - Tracking the movements of fish, edible } \\
\text { seaweed, shellfish etc. } \\
\text { - Also, the ocean: Tides, lunar cycle, } \\
\text { salinity, possibly the currents at Port } \\
\text { Waikato, Whaingaroa, and Kawhia } \\
\end{array}$ \\
\hline $\begin{array}{l}\text { Waipuna- } \\
\text { aa-rangi }\end{array}$ & $\begin{array}{c}\text { Water that pools in } \\
\text { the sky }\end{array}$ & $\begin{array}{l}\text { - Acoustic rain gauge - measures the } \\
\text { sound of water droplets hitting surfaces } \\
\text { (doesn't emphasise rain over mist) } \\
\text { - Humidity }\end{array}$ \\
\hline $\begin{array}{c}\text { Tupu-aa- } \\
\text { nuku }\end{array}$ & $\begin{array}{l}\text { That which grows } \\
\text { in the ground }\end{array}$ & $\begin{array}{l}\text { - Vibrations of root growth } \\
\text { - pH, moisture, temperature etc. } \\
\text { - Vibrations of the ground? }\end{array}$ \\
\hline $\begin{array}{l}\text { Tupu-aa- } \\
\text { rangi }\end{array}$ & $\begin{array}{l}\text { That which grows } \\
\text { in the sky }\end{array}$ & $\begin{array}{l}\text { - Tracking the movements and sounds of } \\
\text { birds } \\
\text { - Growth and movement of tree branches }\end{array}$ \\
\hline Ururangi & $\begin{array}{l}\text { Connected to the } \\
\text { wind }\end{array}$ & $\begin{array}{l}\text { - Wind speed and direction } \\
\text { - Temperature } \\
\text { - Pressure etc. }\end{array}$ \\
\hline
\end{tabular}

\section{B. Proposed Design}

The selection of sensors will be based on their specifications and these selected sensors should be compatible with the other hardware. The sensors are also selected based on their performance in outdoor environment. Since this project is being enabled by the Hamilton city council, there is a stringent requirement only to select sensors which will not pollute the environment with any harmful chemicals or otherwise adversely affect flora or fauna. For instance, the decision to use thermalimaging camera sensors instead of ultrasonic sensors was informed by the possibility of interfering with the navigation capabilities of a nearby bat population. The proposed wireless sensor network for Waka is shown below in figure 5 .
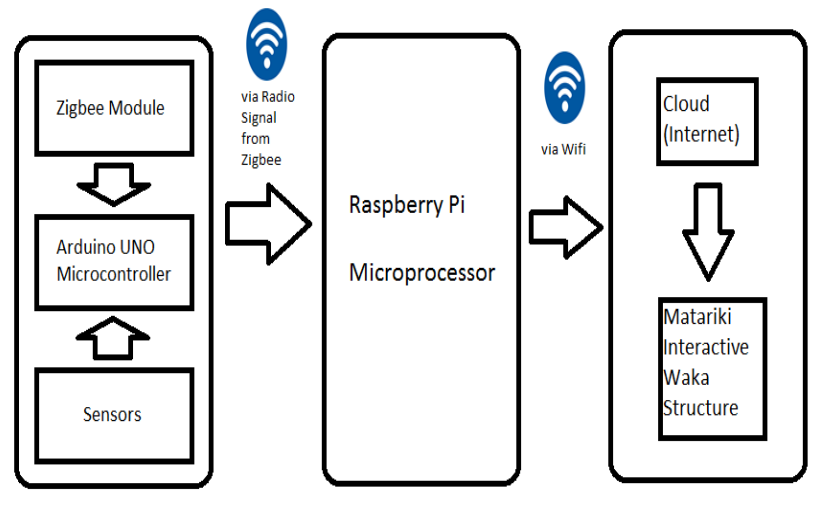

Figure 5. Block diagram of the proposed WSN design 


\section{CONCLUSION}

The aim of this research is to design a 6-metre interactive waka sculpture inspired by Matariki and made out of corten plate steel, with various wireless sensors installed. The interactive aspects will be informed by environmental sensor networks that relate to each individual domain of the Matariki stars, as well as motion tracking sensors on the waka sculpture. By collaboration between technologists, engineers and artists this research aims to design, build new forms of interactive complex artworks. The role of a smart interactive art structure, design methodology and the novel ways in which the interactive structure can serve the intended purpose is discussed

\section{REFERENCES}

[1] J. Sheridan and N. Bryan-Kinns, "Designing for Performative Tangible Interaction.," International Journal of Arts and Technology, vol. 1, no. 3, pp. 288308, 2008.

[2] M. Helmuth, J. H. Jun, T. Oliveira, J. B and K. Merkowitz, "Wireless Sensor Networks and Computer Music, Dance and Installation Implementations," in In Proceedings of the International Computer Music Conference, San Francisco, 2010.

[3] N. Rasamimanana, "Modular Musical Objects Towards Embodied Control Of Digital Music Real Time Musical Interactions," in In Proceedings of the fifth international conference on Tangible, embedded, and embodied interaction, 2011.

[4] M. Feldmeier and J. Paradiso, "An Interactive Music Environment for Large Groups with Giveaway Wireless Motion Sensors," Computer Music Journal, vol. 31, no. 1, pp. 50-67, 2007.

[5] T. Winkler, "Participation and Response in Movement sensing Installations," in In Proceedings of International Computer Music Conference, Chicago, 2000. 